\title{
Magnetization of GaAs Parabolic Quantum Dot by Variation Method
}

\author{
Ayham SHAER, Mohammad K. ELSAID and Musa ELHASAN \\ Physics Department, Faculty of Science, An-Najah National University, Nablus, West Bank, Palestine
}

\begin{abstract}
The magnetization of two interacting electrons confined in a quantum dot presented in a magnetic field had been calculated by solving the relative Hamiltonian using variational method. We had investigated the dependence of the magnetization on temperature, magnetic field strength and confining frequency. The singlet-triplet transitions in the ground state of the quantum dot spectra and the corresponding jumps in the magnetization curves had been shown. The comparisons show that our results are in very good agreement with reported works.
\end{abstract}

Key words: Magnetization, variational method, quantum dot.

\section{Introduction}

QDs (Quantum dots), or artificial atoms, had been the subject of interest research due to its physical properties and great potential device applications such as quantum dot lasers, solar cells, single electron transistors and quantum computers [1-5]. The application of a magnetic field perpendicular to the dot plane will introduce an additional structure on the energy levels and correlation effects of the interacting electrons confined in a quantum dot.

Different approaches had been used to solve the two electrons QD Hamiltonian, including the effect of an applied magnetic field, to obtain the eigenenergies and eigenstates of the QD-system. Wagner, Merkt and Chaplik [6] had studied this interesting QD system and predicted the oscillations between spin-singlet (S) and spin-triplet $(\mathrm{T})$ ground states.

Taut [7] had managed to obtain the exact analytical results for the energy spectrum of two interacting electrons through a coulomb potential, confined in a QD, just for particular values of the magnetic field strength. In Refs. [8, 9] the authors had solved the QD-Hamiltonian by variational method and obtained

Corresponding author: Mohammad K. ELSAID, professor, research fields: nanoscience, nanotechnology. the ground state energies for various values of magnetic field $\left(\omega_{c}\right)$, and confined frequency $\left(\omega_{0}\right)$. In addition, they had performed exact numerical diagonalization for the Helium QD-Hamiltonian and obtained the energy spectra for zero and finite values of magnetic field strength. Kandemir [10, 11] had found the closed form solution for this QD Hamiltonian and the corresponding eigenstates for particular values of the magnetic field strength and confinement frequencies. Elsaid [12-16] had used the dimensional expansion technique, in different works, to study and solve the QD-Hamiltonian and obtain the energies of the two interacting electrons for any arbitrary ratio of coulomb to confinement energies and gave an explanation to the level crossings.

Maksym and Chakraborty [17] had used the diagonalization method to obtain the eigenenergies of interacting electrons in a magnetic field and show the transitions in the angular momentum of the ground states. They had also calculated the heat capacity curve for both interacting and non-interacting confined electrons in the QD presented in a magnetic field. The interacting model shows very different behavior from non-interacting electrons, and the oscillations in these magnetic and thermodynamic quantities like magnetization $(\mathcal{M})$ and heat capacity 
$\left(C_{\mathrm{v}}\right)$ are attributed to the spin singlet-triplet transitions in the ground state spectra of the quantum dot. De Groote, Hornos and Chaplik [18] had also calculated the magnetization, susceptibility and heat capacity of helium like confined QDs and obtained the additional structure in magnetization. In a detailed study, Nguyen and Peeters [19] had considered the QD helium in the presence of a single magnetic ion and applied magnetic field taking into account the electron-electron correlation in many quantum dot. They had shown the dependence of these thermal and magnetic quantities: $\mathrm{C}_{\mathrm{v}}, \mathcal{M}$ and $\chi$ on the strength of the magnetic field, confinement frequency, magnetic ion position and temperature. They had observed that the cusps in the energy levels show up as peaks in the heat capacity and magnetization. In Ref. [20], the authors had used the SFA (static fluctuation approximation) to study the thermodynamic properties of two dimensional GaAs/AlGaAs parabolic QD in a magnetic field.

Boyacioglu and Chatterjee [21] had studied the magnetic properties of a single quantum dot confined with a Gaussian potential model. They observed that the magnetization curve shows peaks structure at low temperature. Helle, Harju and Nieminen [22] had computed the magnetization of a rectangular QD in a high magnetic field and the results show the oscillation and smooth behavior in the magnetization curve for both, interacting and non-interacting confined electrons, respectively.

In an experimental work [23], the magnetization of electrons in GaAs/AlGaAs semiconductor QD as function of applied magnetic field at low temperature $0.3 \mathrm{~K}$ had been measured. They had observed oscillations in the magnetization. To reproduce the experimental results of the magnetization, they found that the electon-electron interaction should be taken into account in the theoretical model of the QD magnetization.

Furthermore, the DFT (density functional method) had been used to investigate the magnetization of a rectangular QD in the applied external magnetic field [24].

Climente, Planelles and Movilla had studied the effect of coulomb interaction on the magnetization of quantum dot with one and two interacting electrons [25].

Very recently, Avetisyan, Chakraborty and Pietilainen [26] had studied the magnetization of anisotropic QD in the presence of the Rashba spin-orbit interaction for three interacting electrons in the dot.

In this work, we had calculated the magnetization as a thermodynamic quantity for a quantum dot helium atom in which both the magnetic field and the electron-electron interaction are fully taken into account. Since, the eigenvalues of the electrons in the QD are the starting point to calculate the physical properties of the QD system [27], we had, first, applied the variational method to solve the QD Hamiltonian and obtain the eigenenergies. The variational calculation method is used as an efficient technique to study the electronic properties of coupled quantum dot [29]. Second, we had used the computed eigenenergies spectra to display theoretically the behavior of magnetization and magnetic susceptibility of the QD as a function of magnetic field strength, confining frequency and temperature.

The rest of this paper is organized as follows: the Hamiltonian theory and computation variational technique of the interacting quantum helium atom are presented in Section 2. In Section 3, we show how to calculate the magnetization and susceptibility from the mean energy expression. Final section will be devoted to numerical results and conclusions.

\section{Theory}

In this section, we will describe in detail the main three parts of the theory, namely: quantum dot Hamiltonian, variational method and the magnetization. 


\subsection{Quantum Dot Hamiltonian}

The effective mass Hamiltonian for two interacting electrons confined in a QD by a parabolic potential in a uniform magnetic field of strength $\mathrm{B}$, applied along $\mathrm{z}$ direction is given by:

$$
\begin{gathered}
\sum_{j=1}^{2}\left\{\frac{1}{2 m^{*}}\left[p\left(r_{j}\right)+\frac{e}{c} A\left(r_{j}\right)\right]^{2}+\frac{1}{2} m^{*} \omega_{0}^{2} r_{j}^{2}\right\}+ \\
\frac{e^{2}}{\epsilon\left|r_{1}-r_{2}\right|}
\end{gathered}
$$

where $\omega_{0}$ and $\epsilon$ are defined as the confining frequency and the dielectric constant for the GaAs medium respectively. $r_{1}$ and $r_{2}$ describe the positions of the first and second electron in the xy plane. $\omega_{c}$ is the cyclotron frequency and the symmetric gauge $\mathrm{A}=\frac{1}{2} \mathrm{~B} \times \mathrm{r}$ is used.

The quantum dot Hamiltonian can be decoupled into center of mass and relative parts by using the standard coordinate transformation.

The center of mass Hamiltonian is a harmonic oscillator type with well-known eigenenergies:

$$
\begin{gathered}
\mathrm{E}_{\mathrm{n}_{\mathrm{cm}}, \mathrm{m}_{\mathrm{cm}}}=\left(2 \mathrm{n}_{\mathrm{cm}}+\left|\mathrm{m}_{\mathrm{cm}}\right|+1\right) \hbar \sqrt{\frac{\omega_{\mathrm{c}}^{2}}{4}+\omega_{\mathrm{o}}^{2}}+ \\
\mathrm{m}_{\mathrm{cm}} \frac{\hbar \omega_{\mathrm{c}}}{2}
\end{gathered}
$$

where $\mathrm{n}_{\mathrm{cm}}, \mathrm{m}_{\mathrm{cm}}$ are the radial and angular quantum numbers respectively.

However, the relative motion Hamiltonian part $\left(\mathrm{H}_{\mathrm{rm}}\right)$, given by the following equation:

$$
\mathrm{H}_{\mathrm{rm}}=\frac{1}{2 \mu}\left[\mathrm{p}_{\mathrm{r}}+\frac{\mathrm{e}}{\mathrm{c}} \mathrm{A}(\mathrm{r})\right]^{2}+\frac{1}{2} \mu \omega_{0}^{2} \mathrm{r}^{2}+\frac{\mathrm{e}^{2}}{\epsilon|\mathrm{r}|}
$$

where $\mu=\frac{m^{*}}{2}$. This Hamiltonian Eq. (3) does not have an analytical solution for all ranges of $\omega_{0}$ and $\omega_{c}$.

\subsection{Varaitional Method Calculations}

Dyblaski, in a recent work [30], had used successfully the variational method to study the electronic structure of the quantum dot. Encouraged by the accuracy of the variational method used in Ref. [28], we shall apply the variational technique to calculate the complete eigenenergy spectra of the QD hamiltonian and the exchange energy $(\mathrm{J})$ as functions of confining frequency and magnetic field strength. In this work, we adopted one-parameter variation wave function as,

$$
\psi(r)=\sqrt[4]{\alpha} \frac{u_{m}(\rho) e^{i m \phi}}{\sqrt{2 \pi} \sqrt{\rho}}
$$

where

$$
\begin{gathered}
u_{m}(\rho)=\rho^{1 / 2+|m|}(1+\beta \rho) e^{-\left(\frac{\rho^{2}}{2}\right)} \\
\rho=\sqrt{\alpha} r
\end{gathered}
$$

All the mathematical steps which lead to the energy expression will be given in details just to make the work self-contained. We can write Schrödinger equation with complete Hamiltonian form and full variational wave function as,

$$
\begin{gathered}
\left(-\frac{\hbar^{2}}{\mathrm{~m}}\left(\mathrm{r}^{-1 / 2} \frac{\partial^{2}}{\partial \mathrm{r}^{2}} \mathrm{r}^{1 / 2}+\frac{1}{\mathrm{r}^{2}}\left(\frac{\partial^{2}}{\partial \phi^{2}}+\frac{1}{4}\right)\right)\right. \\
+4 \mathrm{mr}^{2} \alpha^{2}-\frac{1}{2} \mathrm{i} \hbar \frac{\partial}{\partial \phi} \omega \\
\left.+\frac{\mathrm{e}^{2}}{\epsilon|\mathrm{r}|}\right) \sqrt[4]{\alpha} \frac{\mathrm{u}_{\mathrm{m}}(\rho) \mathrm{e}^{\mathrm{im} \phi}}{\sqrt{2 \pi} \sqrt{\rho}} \\
=\mathrm{E}_{\mathrm{r}} \sqrt[4]{\alpha} \frac{\mathrm{u}_{\mathrm{m}}(\rho) \mathrm{e}^{\mathrm{im} \phi}}{\sqrt{2 \pi} \sqrt{\rho}}
\end{gathered}
$$

In our calculations, we have used the following Atomic Rydberg units

$$
e^{2}=2, \hbar=1, m=1, \epsilon=1
$$

Finally, the equation for a relative coordinate part is

$$
\begin{gathered}
-2 \frac{d^{2}}{d r^{2}}+2\left(\mathrm{~m}^{2}-\frac{1}{4}\right) \frac{1}{\mathrm{r}^{2}}+\frac{1}{2} \omega_{c} m \\
+2 r^{2} \alpha^{2}+\frac{2}{r}
\end{gathered}
$$

We have normalized our wave function,

$$
u_{m}(\rho)=C_{m} \rho^{1 / 2+|m|}(1+\beta \rho) e^{-\left(\frac{\rho^{2}}{2}\right)}
$$

by calculating the normalizing constant as,

$$
C_{m}^{2}=\frac{2 \sqrt{\alpha}}{\left(1+\beta^{2}\right) \Gamma[1+|\mathrm{m}|]+\beta^{2}|\mathrm{~m}| \Gamma[1+|\mathrm{m}|]+2 \beta \Gamma\left[\frac{3}{2}+|\mathrm{m}|\right]}
$$


The above normalization constant can be rewritten in terms of new constant parameters,

where

$$
\mathrm{C}_{\mathrm{m}}^{2}=\frac{\sqrt{\alpha}}{d+e \beta+f \beta^{2}}
$$

$$
\begin{gathered}
\mathrm{d}=\frac{1}{2 \Gamma[1+|\mathrm{m}|]} \quad \begin{array}{r}
\text { We have found the energy spectra of the rel } \\
\text { Hamiltonian in a closed analytical form: }
\end{array} \\
\frac{1}{2} \omega_{\mathrm{c}} \mathrm{m}+\frac{\mathrm{C}_{\mathrm{m}}^{2} \times 2 \alpha}{\sqrt{\alpha}}\left(\frac{1}{2} \mathrm{~m}^{2} \Gamma[|\mathrm{m}|]+\frac{1}{2}|\mathrm{~m}| \Gamma[|\mathrm{m}|]+\frac{5}{8} \beta^{2}|\mathrm{~m}| \Gamma[|\mathrm{m}|]+\frac{1}{2} \mathrm{~m}^{2} \beta^{2} \Gamma[|\mathrm{m}|]+\frac{\Gamma\left[\frac{1}{2}+|\mathrm{m}|\right]}{2 \sqrt{\alpha}}\right. \\
-\frac{1}{4} \beta \Gamma\left[\frac{1}{2}+|\mathrm{m}|\right]+\mathrm{m}^{2} \beta \Gamma\left[\frac{1}{2}+|\mathrm{m}|\right]+\frac{\beta^{2} \Gamma\left[\frac{1}{2}+|\mathrm{m}|\right]}{4 \sqrt{\alpha}}+\frac{\beta^{2}|\mathrm{~m}| \Gamma\left[\frac{1}{2}+|\mathrm{m}|\right]}{2 \sqrt{\alpha}} \\
+\frac{\beta \Gamma[1+|\mathrm{m}|]}{\sqrt{\alpha}}-\frac{1}{8} \beta^{2} \Gamma[1+|\mathrm{m}|]+\frac{1}{2} \mathrm{~m}^{2} \beta^{2} \Gamma[1+|\mathrm{m}|]+\beta \Gamma\left[\frac{3}{2}+|\mathrm{m}|\right] \\
\left.+\frac{1}{2} \Gamma[2+|\mathrm{m}|]+\beta^{2} \Gamma[2+|\mathrm{m}|]+\frac{1}{2} \beta^{2}|\mathrm{~m}| \Gamma[2+|\mathrm{m}|]+\beta \Gamma\left[\frac{5}{2}+|\mathrm{m}|\right]\right)
\end{gathered}
$$

which can be rewritten as,

$$
E_{r}(\beta)=-\frac{1}{2} m \omega_{\mathrm{c}}+2 \alpha \frac{a+b \beta+c \beta}{d+e \beta+f \beta}
$$

where

$$
\begin{gathered}
\mathrm{a}=\frac{\mathrm{e}}{(2|\mathrm{~m}|+1) \sqrt{\alpha}}+2 \mathrm{f} \\
\mathrm{b}=\frac{2 \mathrm{~d}}{\sqrt{\alpha}}+2(|\mathrm{~m}|+1) \mathrm{e} \\
\mathrm{c}=\frac{\mathrm{e}}{2 \sqrt{\alpha}}+\left(2|\mathrm{~m}|^{2}+4|\mathrm{~m}|+3\right) \mathrm{d}
\end{gathered}
$$

The parameters: $d$, e, f which are defined previously in Eqs. (12)-(14) respectively.

The energy eigenvalues of $H_{r}$ can be obtained by minimizing the energy expression eq. (16) with respect to the variational parameter $\beta$ namely,

$$
\frac{\partial E}{\partial \beta}=0, \frac{\partial^{2} E}{\partial \beta^{2}}>0
$$

The value of the parameter $\beta$ which satisfies the minimum energy requirement is,

$$
\beta_{\text {min }, m}=\frac{2 c d-2 a f-\sqrt{(2 c d-2 a f)^{2}-4(b d-a e)(c e-b f)}}{2(-c e+b f)}
$$

So, the final energy expression in terms of the variational parameter value which satisfies the minimization condition is

$$
\begin{aligned}
E_{r}\left(\beta_{\min }\right)=-\frac{1}{2} & m \omega_{\mathrm{c}} \\
& +2 \alpha \frac{a+b \beta_{\min }+c \beta_{\min }^{2}}{d+e \beta_{\min }+f \beta_{\min }^{2}}
\end{aligned}
$$

Having obtained the eigenenergies for the QD system for any state labeled by $n, m$ quantum number, we are able to calculate the exchange energy (J) define as:

$$
J=E_{\text {triplet }}-E_{\text {singlet }}
$$

for any range of a magnetic field and confining potential.

\subsection{Magnetization of the Quantum Dot}

The magnetization of the QD system is evaluated as the magnetic field derivative of the mean energy of the QD. 


$$
M\left(T, B, \omega_{0}\right)=\frac{-\partial\left\langle\mathrm{E}\left(\mathrm{T}, \mathrm{B}, \omega_{0}\right)\right\rangle}{\partial \mathrm{B}}
$$

where the statistical average energy is calculated as:

$$
\left\langle\mathrm{E}\left(\mathrm{T}, \mathrm{B}, \omega_{0}\right)\right\rangle=\frac{\sum_{\alpha=1}^{\mathrm{N}} \mathrm{E}_{\alpha} \mathrm{e}^{-\mathrm{E}_{\alpha} / \mathrm{k}_{\mathrm{B}} \mathrm{T}}}{\sum_{\alpha=1}^{\mathrm{N}} \mathrm{e}^{-\mathrm{E}_{\alpha} / \mathrm{k}_{\mathrm{B}} \mathrm{T}}}
$$

and the sum is taken over energy levels of the QD.

The dependence of the computed magnetization on the magnetic field $\omega_{c}$, confining frequency $\left(\omega_{0}\right)$ and temperature $(\mathrm{T})$ will be displayed.

\section{Results and Conclusions}

The results for two interacting electrons in a quantum dot made from GaAs material (effective Rydberg Ry*= $5.825 \mathrm{meV}$ ) are presented in Figs. 1 to 5. In Fig. 1, we had displayed our computed energy spectra results for two interacting electrons confined parabolically in a QD as a function of the strength of the magnetic field for various values of angular momentum ( $\mathrm{m}$ ) and fixed value of confining frequenc $\omega_{0}=\frac{2}{3}$ yRy*. The comparison with Ref. [30] clearly shows excellent agreement between both works.
Fig. 1 shows the transition in the angular momentum of the ground state of the QD system as the magnetic field increases. The origin of these transitions is due to the effect of coulomb interaction energy in the QD Hamiltonian. These transitions in the angular momentum of the QD system correspond to the (S-T) transitions manifest themselves as cusps in the magnetization curve of the QD. In Fig. 2, we had shown the dependence of the magnetization on the magnetic field strength for fixed values of the confining frequency $\omega_{0}=\frac{2}{3} R^{*}$ and $\mathrm{T}=0.01 \mathrm{~K}$, for both interacting and non-interacting electrons in a QD. The non-interacting curve (solid line) shows a smooth behavior while the interacting curve (dashed line) shows a saw tooth behavior. The magnetization shows a peak structure which is a result of the transition in the angular momentum of the ground state energy as shown and discussed previously. For example, the first peak corresponds to the transition in the angular momentum of the ground state from: $m_{r}=0$ $\operatorname{tom}_{\mathrm{r}}=1$.

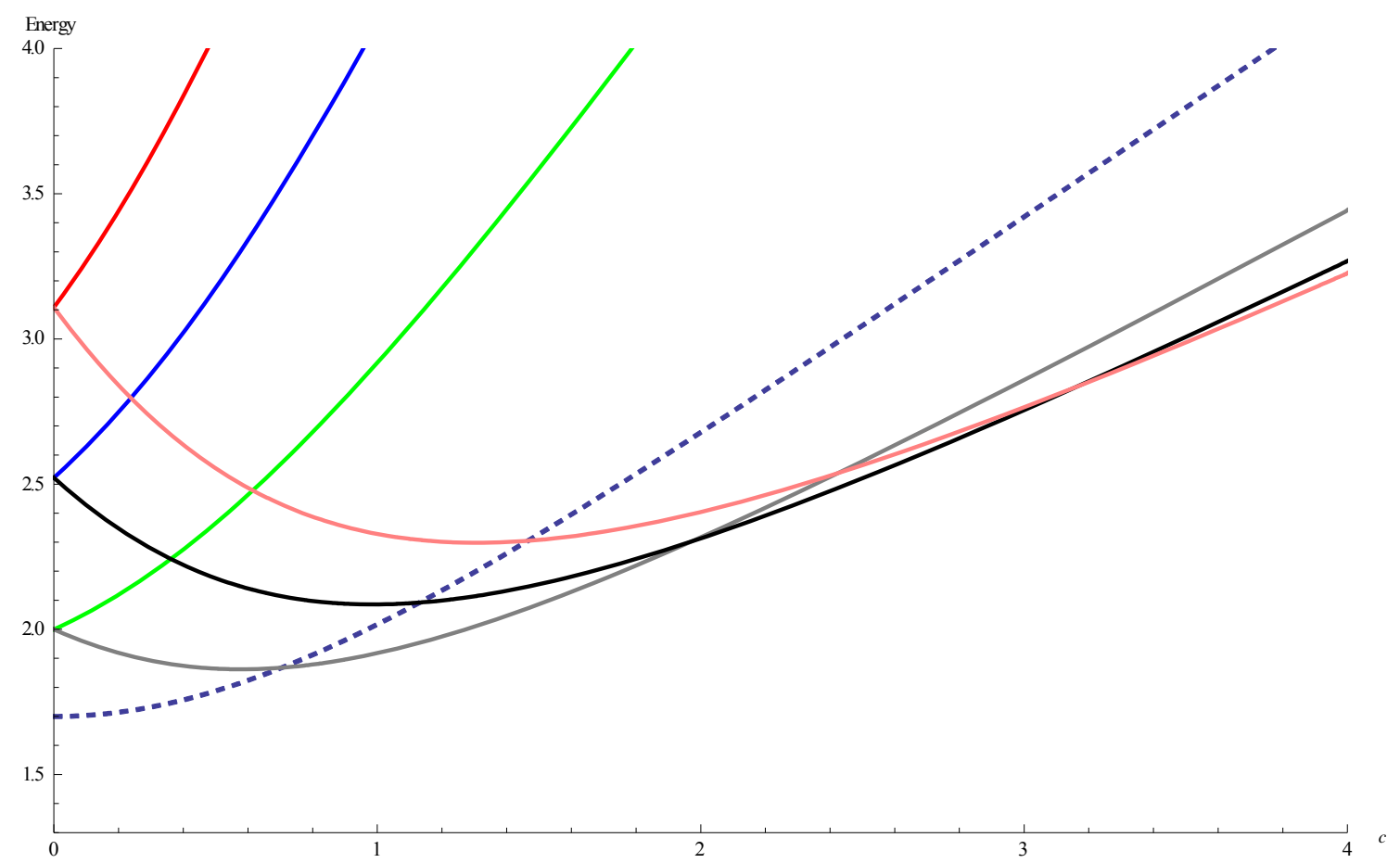

Fig. 1 The computed results of two interacting electron quantum dot against the strength of the magnetic field for $\omega_{0}=$ $\frac{2}{3}$ Ry*. 


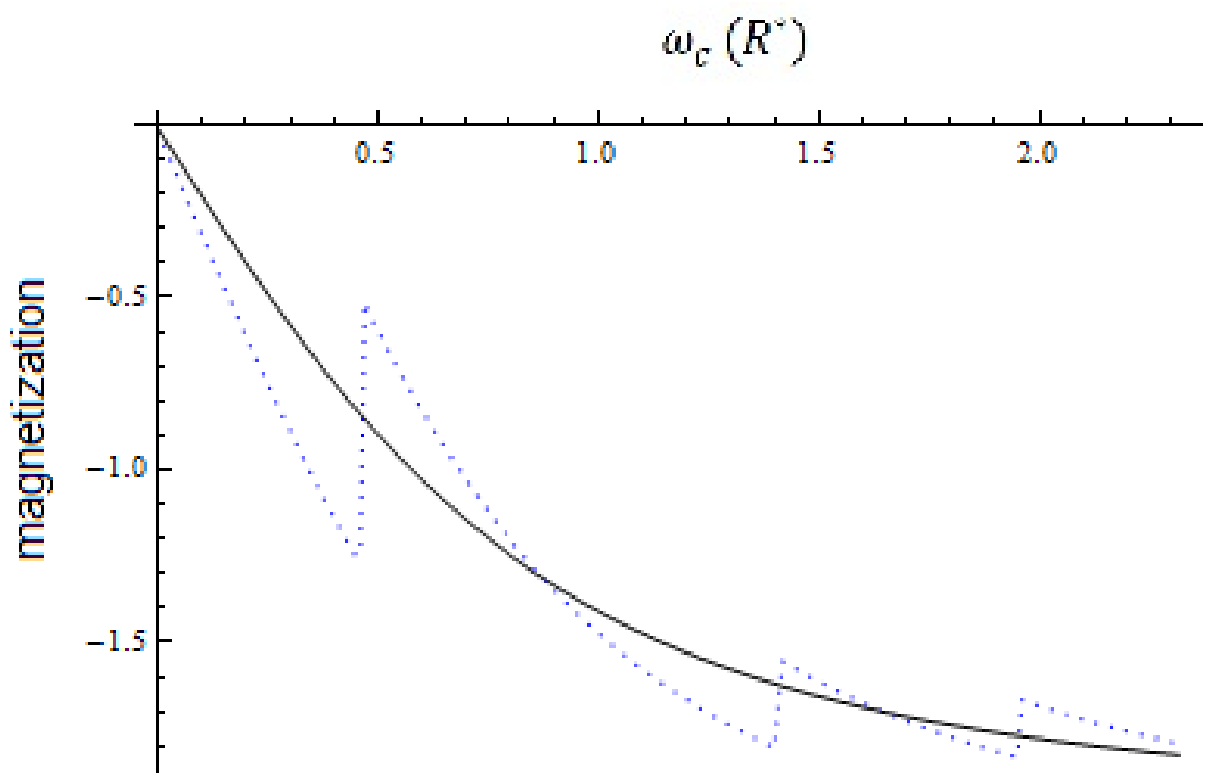

Fig. 2 The magnetization of two electrons quantum dot against the magnetic field strength for non-interacting (solid line) and interacting (dashed) cases calculated at $\mathrm{T}=0.01 \mathrm{~K}$ and confinement frequency $\left(\frac{2}{3} \mathrm{Ry} *\right)$.

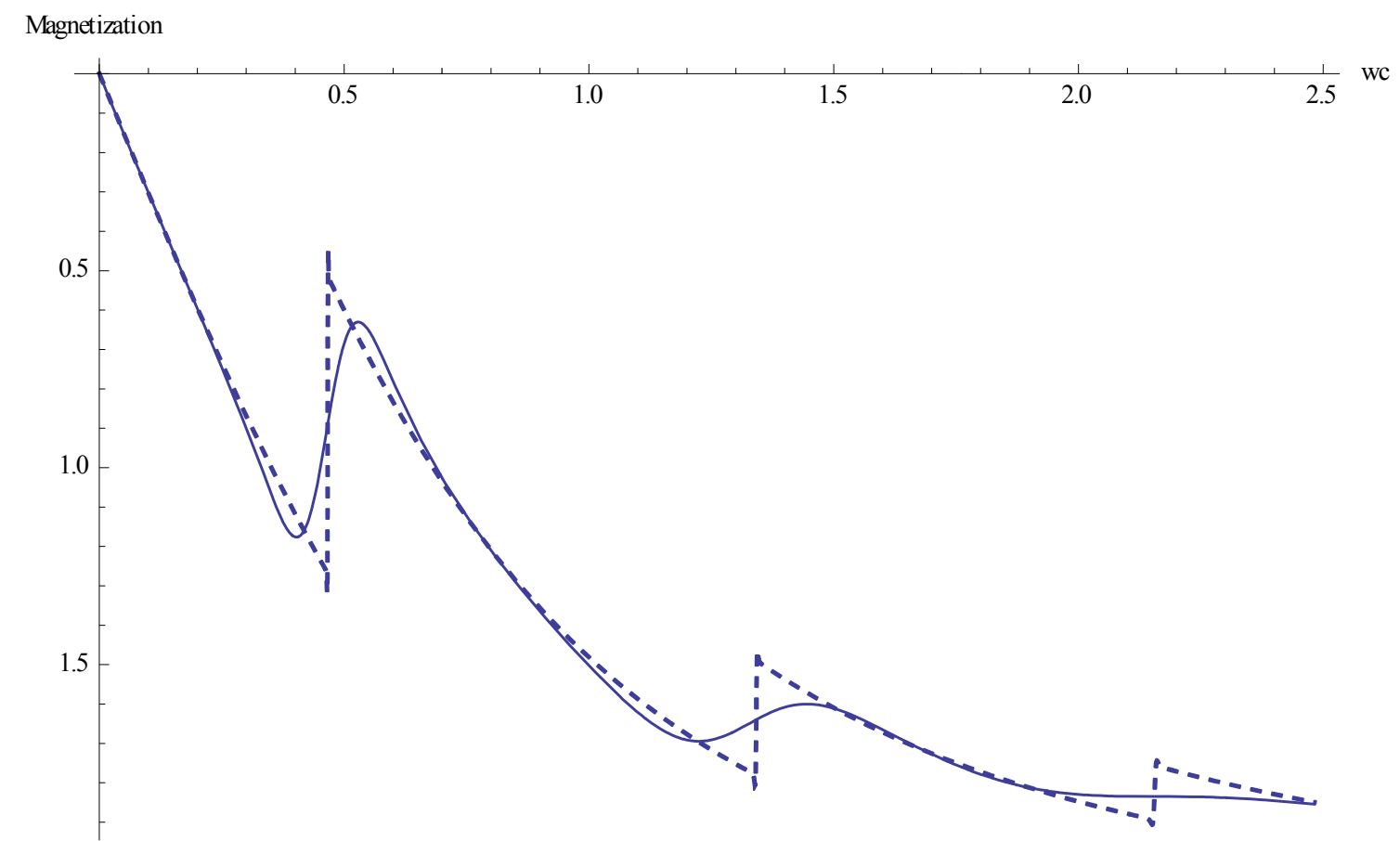

Fig. 3 The behavior of the magnetization of the two interacting electrons quantum dot as function of magnetic field strength for fixed value of confining frequency $\left(\omega_{0}=\frac{1}{2} R y^{*}\right)$ and various $T$ : $(T=0.01 K$ dashed and $T=1 \mathrm{~K} 1$ solid). 


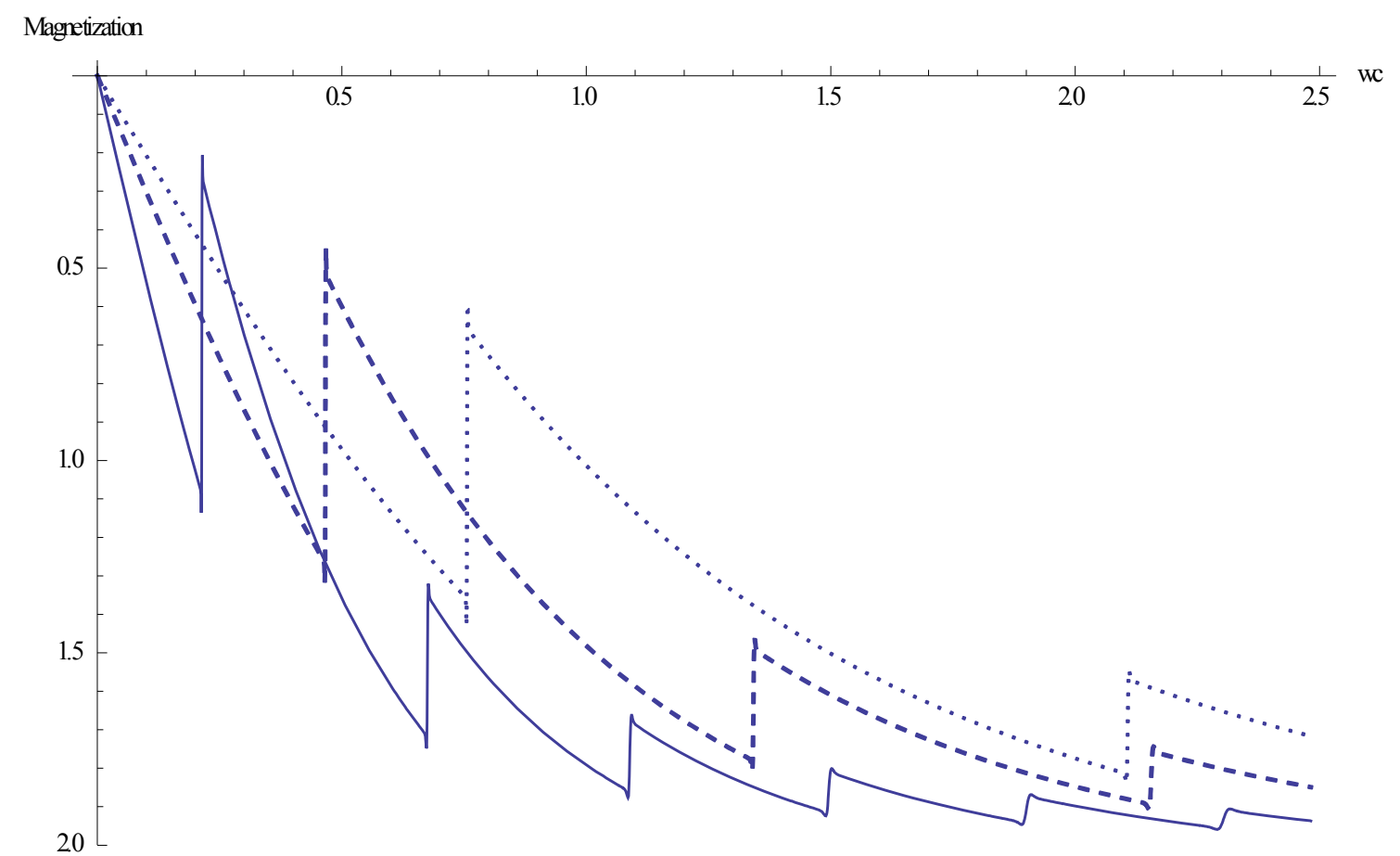

Fig. 4 The magnetization of two interacting electrons quantum dot as function of magnetic field strength at $\mathrm{T}=\mathbf{0 . 0 1} \mathrm{K}$ and various confinement frequencies $\omega_{0}$ : $\left(\omega_{0}=0.3\right.$ solid; $\omega_{0}=0.5$ dashed and $\omega_{0}=0.7$ dotted $)$.

In Fig. 3, we had shown the behavior of the magnetization against the magnetic field strength at fixed value of confining frequency $\omega_{0}=\frac{2}{3} R^{*}$ and different temperature values $\mathrm{T}=0.01 K$ (dashed curve) and $1.0 \mathrm{~K}$ (solid curve). It is observed that the heights of the peak due to transition jumps are reduced, broadened and shifted to higher magnetic value as the temperature increased.

In Fig. 4, we had shown the effect of confinement frequency on the magnetization curve, while the temperature is kept unchanged. The curve clearly shows the gradual shift of the magnetization jumps to higher magnetic field as the confining frequency $\omega_{0}$ enhanced. This behavior for the magnetization is in agreement with the results of Refs. [21, 25].

In conclusion, we had applied the variational method to solve the Hamiltonian for two interacting electrons confined parabolically in a quantum dot subjected to a magnetic field in order to study theoretically the details of the dependence of the magnetization on the magnetic field, confining frequency and temperature of the QD system. The investigations clearly show that the oscillations in magnetization are due to the role of electron-electron interaction, as reported in previous works.

\section{References}

[1] Ashoori, R. C., Stormer, H. L., Weiner, J. S., Pfeiffer, L. N., Baldwin, K. W., and West, K. W. 1993. "N-Electron Ground State Energies of a Quantum Dot in Magnetic Field." Phys. Rev. Let. 71 (4): 613-16.

[2] Ciftja, O. 2013. "Understanding Electronic Systems in Semiconductor Quantum Dots.” Physica Scripta 88 (5): 058302.

[3] Kastner, M. A. 1992. "The Single-Electron Transistor." Rev. Mod. Phys. 64: 849.

[4] Loss, D., and Divincenzo, D. P. 1998. "Quantum Computation with Quantum Dots.” Phys. Rev. A 57: 120.

[5] Burkard, G., Loss, D., and Divincenzo, D. P. 1999. "Coupled Quantum Dots as Quantum Gates." Phys. Rev. B 59: 2070-8.

[6] Wagner, M., Merkt, M. U., and Chaplik, A. V. 1992. "Spin-Singlet-Spin-Triplet Oscillations in Quantum Dots." Phys. Rev. B 45: 1951.

[7] Taut, M. 1994. "Two Electrons in a Homogeneous Magnetic Field: Particular Analytical Solutions.” J. Phys. A: Math. Gen. 27 (13): 1045. 
[8] Ciftja, O., and Kumar, A. A. 2004. "Ground State of Two-Dimensional Quantum-Dot Helium in Zero Magnetic Field: Perturbation, Diagonalization, and Variational Theory." Phys. Rev. B 70 (20): 205326.

[9] Ciftja, O., and Faruk, M. G. 2005. "Two-Dimensional Quantum-Dot Helium in a Magnetic Field: Variational Theory." Phys. Rev. B 72 (20): 205334.

[10] Kandemir, S. 2005. "Variational Study of Two-Electron Quantum Dots.” Phys. Rev. B 72 (16): 165350.

[11] Kandemir, S. 2005. "Two Interacting Electrons in a Uniform Magnetic Field and a Parabolic Potential: The General Closed-Form Solution.” J. Math. Phys. 46 (3): 032110 .

[12] El-said, M. 2000. "Spectroscopic Structure of Two Interacting Electrons in a Quantum Dot by the Shifted 1/N Expansion Method.” Phys. Rev. B 61: 13026.

[13] El-said, M. 1995. "Two-Electron Quantum Dots in a Magnetic Field." Semiconductor Sci. Technol. 10 (10): 1310.

[14] El-said, M. 1998. "Two Interacting Electrons in Quantum Dot.” Superlattices and Microstructures 23: 1237.

[15] Elsaid, M. K., Al-Naafa, M. A., and Zugail, S. J. 2008. "Spin Singlet-Triplet Energy Splitting in the Ground State of a Quantum Dot with a Magnetic Field: Effect of Dimensionality." Comput. Theor. Nanosci. 5 (4): 677-80.

[16] El-said, M. 2002. "The Spectral Properties of Two Interacting Electrons in a Quantum Dot." Turkish J. Physics 26: 331.

[17] Maksym, P. A., and Chakraborty, T. 1990. "Quantum Dots in a Magnetic Field: Role of Electron-Electron Interactions." Phys. Rev. Lett. 65 (1): 108.

[18] De Groote, J. J. S., Hornos , J. E. M., amd Chaplik, A.V. 1992. "Thermodynamic Properties of Quantum Dots in a Magnetic Field." Phys. Rev. B 46 (19): 12773.

[19] Nguyen, N., and Peeters, F. M. 2008. "Magnetic Field Dependence of the Many-Electron States in a Magnetic Quantum Dot: The Ferromagnetic-Antiferromagnetic
Transition.” Phys. Rev. B 78 (4): 045321.

[20] Nammas, F. S., Sandouqa, A. S., Ghassib, H. B., and Al-Sugheir, M. K. 2011. "Thermodynamic Properties of Two-Dimensional Few-Electrons Quantum Dot Using the Static Fluctuation Approximation.” Physica B 406 (24): 4671-7.

[21] Boyacioglu, B., and Chatterjee, A. 2012. "Heat Capacity and Entropy of a GaAs Quantum Dot with Gaussian Confinement." J. Appl. Phys. 112 (8): 083514.

[22] Helle, M., Harju, A., and Nieminen, R. M. 2005. "Two-Electron Lateral Quantum-Dot Molecules in a Magnetic Field.” Phys. Rev. B 72 (20): 205329.

[23] Schwarz, M. P., Grundler, D., Wilde, M., and Heyn, Ch., Heitmann, D. 2002. "Magnetization of Semiconductor Quantum Dots.” J. Appl. Phys. 91: 6875.

[24] Räsänen, E., Saarikoski, H., Stavrou, V. N., Harju, A., Puska, M. J., and Nieminen, R. M. 2003. "Electronic Structure of Rectangular Quantum Dots.” Phys. Rev. B 67 (23): 235307.

[25] Climente, J. I., Planelles, J., and Movilla, J. L. 2004. "Magnetization of Nanoscopic Quantum Rings and Dots." Phys Rev. B 70 (8): 081301.

[26] Avetisyan, S., Chakraborty, T., and Pietiläinen, P. 2015. "Magnetization of Quantum Dots: A Measure of Anisotropy and the Rashba Interaction." Cornell University Library. http://arxiv.org/abs/1501.01025.

[27] Fock, V. 1928. "Bemerkung Zur Quantelung Des Harmonischen Oszillators im Magnetfeld.” Z. Phys. 47: 446.

[28] Darwin, C. G. 1930. "The Diamagnetism of the Free Electron.” Math. Proc. Cambridge Phill. Soc. 27: 86.

[29] Nguyen, N. T. T., and Das Sarma, S. 2011. "Impurity Effects on Semiconductor Quantum Bits in Coupled Quantum Dots." Phys. Rev. B 83: 235322.

[30] Dybalski, W., and Hawrylak, P. 2005. "Two Electrons in a Strongly Coupled Double Quantum Dot: from an Artificial Helium Atom to a Hydrogen Molecule.” Phys. Rev. B 72: 205432. 